\title{
A simple transportable imaging system for fast thermal non-destructive testing
}

\author{
by J. VARIS, J. HARTIKAINEN, R. LEHTINIEMI \\ and M. LUUKKALA (*)
}

(") Department of Physics, University of Helsinki, Siltavuorenpenger 20 D, SF-00170 Helsinki, Finland.

\begin{abstract}
A transportable thermal imaging system based on a simple infrared line scanner is described. Either a line focused laser beam or radio frequency induction coil can be used for heating. A carbon-glass fibre composite plate having artificial defects made with Brinell and Vickers indentors was inspected in order to demonstrate the performance of the system. Examples of the results are presented.
\end{abstract}

\section{Introduction}

In practical NDT applications, the inspection time usually has an important role. A lot of effort has concentrated on reducing the measurement time in order to develop fast testing equipment. Photothermal techniques using infrared (IR) cameras or scanners are most promising in this respect.

During the past couple of years we have developed two photothermal techniques that utilize a simple infrared line scanner. The first one uses a line focused high power laser beam for heating [ 1 and 2]. This method has proved to be very suitable for inspecting plasma-sprayed coatings on steel substrates. In the second method radio frequency (RF) induction heating is used [3]. The advantage of this method is the selectivity of the heating. This feature is very practical with composite materials consisting of both conductive and dielectric materials.

In cases when the inspected object cannot be brought to the measurement system for testing, a different approach have to be taken. Instead of moving the object, the scanner can be moved. Constructing a compact hand held line scanner the whole measurement equipment can be divided to two units: the scanner and a control unit consisting of a microcomputer and the needed electronics for driving the system [4]. This way the control unit can be stationary, only the scanner unit needs to be moved. The measurement time is fast enough for the movement to be made with hand. Also the operator can now move around the object. This capability is often needed in applications of maintenance, etc.

\section{Transportable measurement system}

\subsection{General description}

A schematic diagram of the measurement system is shown in figure 1. The system consists of a remote control unit and a hand held scanner unit that is brought to the object to be tested. The sample is heated with a line source perpendicular to the direction of the scanner head movement. The surface temperature of the inspected object is monitored with an infrared line scanner consisting of a liquid nitrogen cooled 


\section{http://dx.doi.org/10.21611/qirt.1992.036}

$\mathrm{HgCdTe}$ detector, a germanium lens and a deflection mirror. The infrared detector is focused on the sample surface with the germanium lens. The surface area of the sample is covered by combining the horizontal scan of the deflection mirror with the vertical movement of the scanner head.

The control unit consists of a microcomputer, a deflection mirror driver amplifier and heat source control electronics. The microcomputer controls the heat source and the line scanner via multifunction $1 / O$ board. It also collects, stores and handles the data obtained with the line scanner. The data is displayed as pseudo-color images on the computer monitor. Basic image enhancement techniques are available. Because of the major role of the computer controlling the whole system, the graphics capabilities and user friendliness were strongly emphasized in design.

\subsection{Laser heating}

In the case of laser heating, the heating beam is brought from the control unit through an optical fibre to the scanner head. The beam is then focused on the object surface on a line using a cylindrical lens with a prism and a mirror (see figure 2). The IR line scanner monitors the surface temperature alongside the heating line. The heating line is $30 \mathrm{~mm}$ in length and $200 \mu \mathrm{m}$ in width. The needed heating power is approximately $10-15 \mathrm{~W}$.

The spatial resolution of the system depends on the resolution of the line scanner and the width of the heating line. With small detector $(25 \times 25 \mu \mathrm{m})$ the laser heating method yields high resolution thermal images. A major disadvantage of the method is the laser itself. High power lasers are often large sized and fragile equipment that need water cooling. Usually this would not encourage the use of such equipment in an industrial environment. The use of an optical fibre allows the laser to be placed in the control unit and thus solves the problem on major parts. The fibre can be several meters in length without causing any significant loss in the heating power. However, the coupling of the light from the laser into the fibre is not necessarily trivial. Especially when the wavelength of the laser light is in infrared region, a visible guidance beam is needed.

\subsection{Radio frequency induction heating}

In the RF induction heating method the line source is produced with a coil (see figure 3.). The length of the coil is $60 \mathrm{~mm}$ and the width $7 \mathrm{~mm}$. The signal for heating is obtained from a signal generator that is connected to a RF amplifier having the maximum output of $200 \mathrm{~W}$. The frequency range is $1 \mathrm{MHz}-30 \mathrm{MHz}$. The signal generator and the amplifier are in the control unit.

As a mechanical arrangement the induction coil is more rugged than the optical system. Because especially the conductive materials can be heated with RF induction, a selective heating is possible, if the tested object consists of both conductive and dielectric materials. This way thermal images with a very high contrast can be produced, although the highly localized laser beam yields a better spatial resolution. Also only a very thin surface layer is heated, because. the induction frequency is high (i.e. small skin depth). The induction coil is a very critical element in the equipment. Making a coil with a suitable resonance frequency with high power transfer capability is somewhat complicated. 


\section{Examples of results}

A carbon-glass fibre composite plate was tested using the RF induction heating method. The plate consists of several layers of carbon fibre nets with glass fibres between the nets. In this case the electrical conductivity of the carbon fibres is higher than that of glass fibres and the effect of selective heating can be applied. Artificial Impact damages were made on the plate with Brinell and Vickers indentors. The loads were $500 \mathrm{~kg}$ with Brinell indentor and $187.5 \mathrm{~kg}$ with Vickers. Figures 4 and 5 show schematically the places of the impacts in respect to the scanned areas. In the thermal Images (figures $A^{*}$ and $B$ ) the impact damages are clearly shown. In the figures the vertical carbon fibres are parallel to the heating coil and thus heat up better than the horizontal ones. In both figures the scanned area is about $60 \mathrm{~mm} \times 30 \mathrm{~mm}$. The size of the defects can be estimated to be $5 \mathrm{~mm} \times 5 \mathrm{~mm}$. The measurement time of one frame is about $6 \mathrm{~s}$.

\section{Conclusions}

We have demonstrated a thermal NDT equipment with reasonably good resolution that can be used in industrial environment. The main emphasis on the design has been the transportability of the equipment. Normally the objects need to be brought to laboratory for testing. Our equipment can be brought to the object, thus making maintenance applications possible. The equipment is simple, fast, inexpensive and user friendly. All of these are attributes that are needed for marketing thermal NDT systems to industry.

\section{REFERENCES}

[1] HARTIKAINEN (J.). - Inspection of plasma-sprayed coatings using fast infrared scanning technique. Rev. Sci. Instrum., 60 (7), 1989, p. 1334-1337.

[2] LEHTINIEMI (R.), HARTIKAINEN (J.), RANTALA (J.), VARIS (J.) and LUUKKALA (M.). - Fast photothermal inspection of plasma-sprayed coatings of primary circulation seal rings of a nuclear reactor. In: Thompson, D.O. \& Chimenti, D.E. (ed.). Review of Progress in Quantitative NDE, vol. 11A, 1992. p. 441-446.

[3] HARTIKAINEN (J.), LEHTINIEMI (R.) and LUUKKALA (M.). - Fast photothermal measurement system utilizing $\mathrm{CO}_{2}$ laser and radio frequency induction heating. In: Thompson, D.O. \& Chimenti, D.E. (ed.). Review of Progress in Quantitative NDE, vol. 10A, 1991. p. 539-543.

[4] VARIS (J.), HARTIKAINEN (J.), LEHTINIEMI (R.) and LUUKKALA (M.). Transportable photothermal NDE equipment for on site measurements. In: Thompson, D.O. \& Chimenti, D.E. (ed.). Review of Progress in Quantitative NDE, vol. 11B, 1992. p. 2257-2260. 
http://dx.doi.org/10.21611/qirt.1992.036

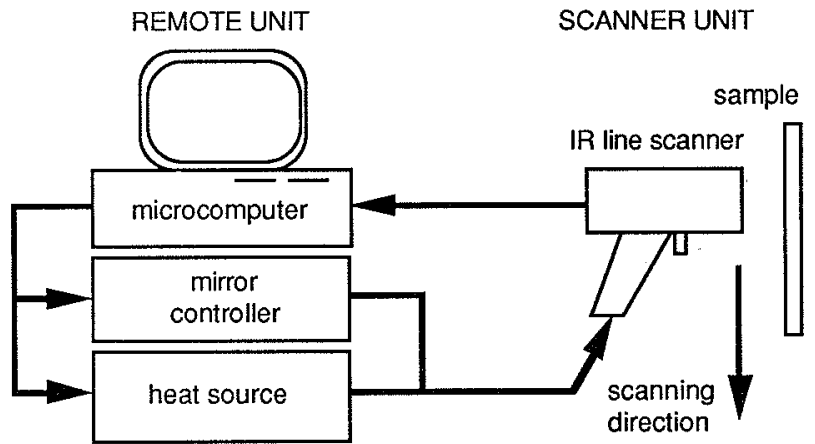

Fig. 1. - Schematic diagram of the transportable imaging system.
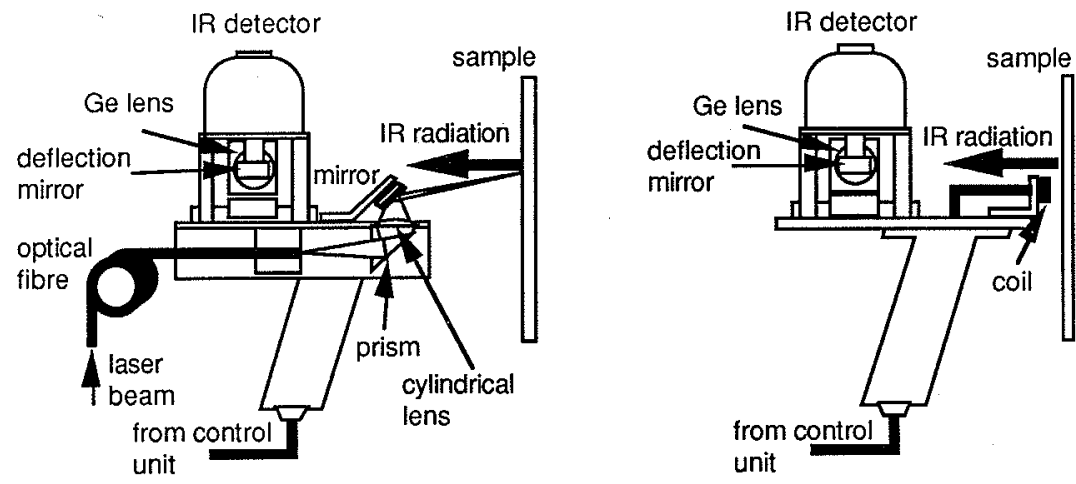

Fig. 2. - Infrared line scanner with laser heating.

Fig. 3. - Infrared line scanner with RF induction heating.

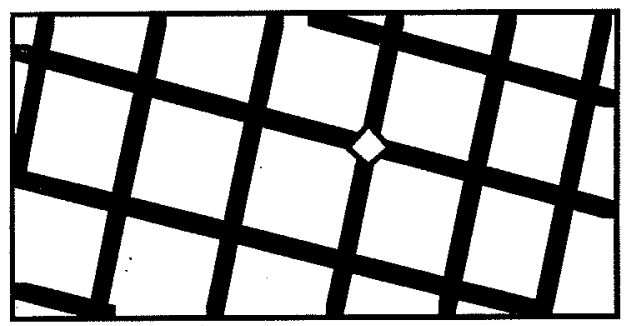

Fig. 4. - Schematic of the carbon fibre net around the Vickers impact.

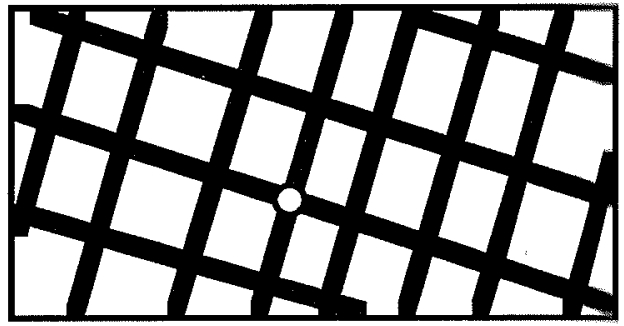

Fig. 5. - Schematic of the carbon fibre net around the Brinell impact. 